\title{
Interpolatory blending net subdivision schemes of Dubuc-Deslauriers type
}

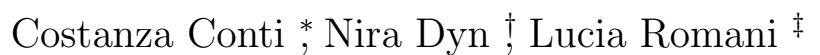

August 15, 2012

\begin{abstract}
Net subdivision schemes recursively refine nets of univariate continuous functions defined on the lines of planar grids, and generate as limits bivariate continuous functions. In this paper a family of interpolatory net subdivision schemes related to the family of Dubuc-Deslauriers interpolatory subdivision schemes is constructed and analyzed. The construction is based on Gordon blending interpolants to nets of univariate functions, and on a particular class of blending functions with properties related to the Dubuc-Deslauriers schemes. The general analysis tools for net subdivision schemes, developed in a previous paper by the authors, together with the properties of the blending functions, lead to the proof of the convergence of these schemes to limit functions having the same integer smoothness as the limits of the corresponding Dubuc-Deslauriers schemes. These results are proved for net subdivision schemes corresponding to the first 84 members of the Dubuc-Deslauriers family, and conjectured for the rest. A concrete example of a family of piecewise polynomial blending functions is considered, together with the corresponding family of net subdivision schemes. The performance of the first two net subdivision schemes in this family is demonstrated by two examples.
\end{abstract}

Key words: Interpolatory net subdivision, Dubuc-Deslauriers interpolatory subdivision, Blending, Proximity, Controllability, Convergence, Smoothness, Z-splines.

2010 Mathematics Subject Classification: 26A15, 26A16, 41A05, 65D05, 65D07, 65D17.

\section{Introduction}

A net subdivision scheme generates limit bivariate functions by repeated refinements of nets of univariate functions defined on planar grids of lines [3, 4, 7]. In [3] a specific approximating net subdivision scheme is constructed and analyzed, while in [7] a specific interpolatory net subdivision scheme is investigated. (A net subdivision scheme is termed interpolatory if the refined net contains the coarser net at all refinement levels, see Figure 1). A family of spline-like net subdivision schemes is presented in [4] and its properties are established by the general tools for the analysis of convergence and smoothness of net subdivision schemes developed in that paper.

Here we present a general construction of families of interpolatory net subdivision schemes. (To the best of our knowledge this is the first family of interpolatory net subdivision schemes to appear in the literature). We analyze these schemes by the general tools in [4], which are based on two important properties of the sequences of generated refined nets; controllability and proximity. In our construction the refined nets are obtained by sampling on refined grids Gordon blending interpolants

\footnotetext{
*Dipartimento di Energetica "Sergio Stecco", Università di Firenze, Viale Morgagni 40/44, 50134 Firenze, Italy. tel.: +39-0554796713 - fax: +39-0554224137 - email: costanza.conti@unifi.it

${ }^{\dagger}$ Dept. of Applied Mathematics, School of Mathematical Sciences, Tel Aviv University, Tel Aviv 69978, Israel. email: niradyn@post.tau.ac.il

${ }^{\ddagger}$ Dipartimento di Matematica e Applicazioni, Università di Milano-Bicocca, Via R. Cozzi 53, 20125 Milano, Italy. tel.: +39-0264485735 - fax: +39-0264485705 - email: lucia.romani@unimib.it
} 
to the coarser nets. For a given scheme, the blending function in the Gordon interpolants is the same at all refinement levels, and has properties which relate it to a corresponding Dubuc-Deslauriers interpolatory scheme refining points [6]. The properties of the blending functions together with the analysis tools of [4], allow us to prove that the so constructed schemes, corresponding to the first 84 members of the Dubuc-Deslauriers family, are convergent and have the integer smoothness of the corresponding Dubuc-Deslauriers schemes. A specific family of blending net subdivision schemes of Dubuc-Deslauriers type is presented, based on blending functions from a family of piecewise polynomial functions known as Z-splines [2, 10].

Our general construction in this paper is limited to regular nets of functions. However, the final goal of our research is the generalization of the proposed refinement algorithms to curve networks containing extraordinary vertices, a setting in which our subdivision approach has significant advantages.

Here is the outline of the paper. In Section 2 net interpolatory subdivision schemes are defined together with the related notions of convergence and smoothness. Also, the notion of controllability and the notion of proximity to a linear, bivariate, interpolatory point subdivision scheme, are recalled from [4]. The family of interpolatory blending net subdivision schemes of Dubuc-Deslauriers type built upon Gordon blending interpolants is considered in Section 3, while the convergence and smoothness analysis of these schemes is presented in Section 4. In Section 5 a specific example of a family of interpolatory blending net subdivision schemes of Dubuc-Deslauriers type based on the Z-splines blending functions is considered. In particular the schemes corresponding to the 4-point and the 6point Dubuc-Deslauriers schemes are presented in more details, and the performance of each scheme is demonstrated by two examples.
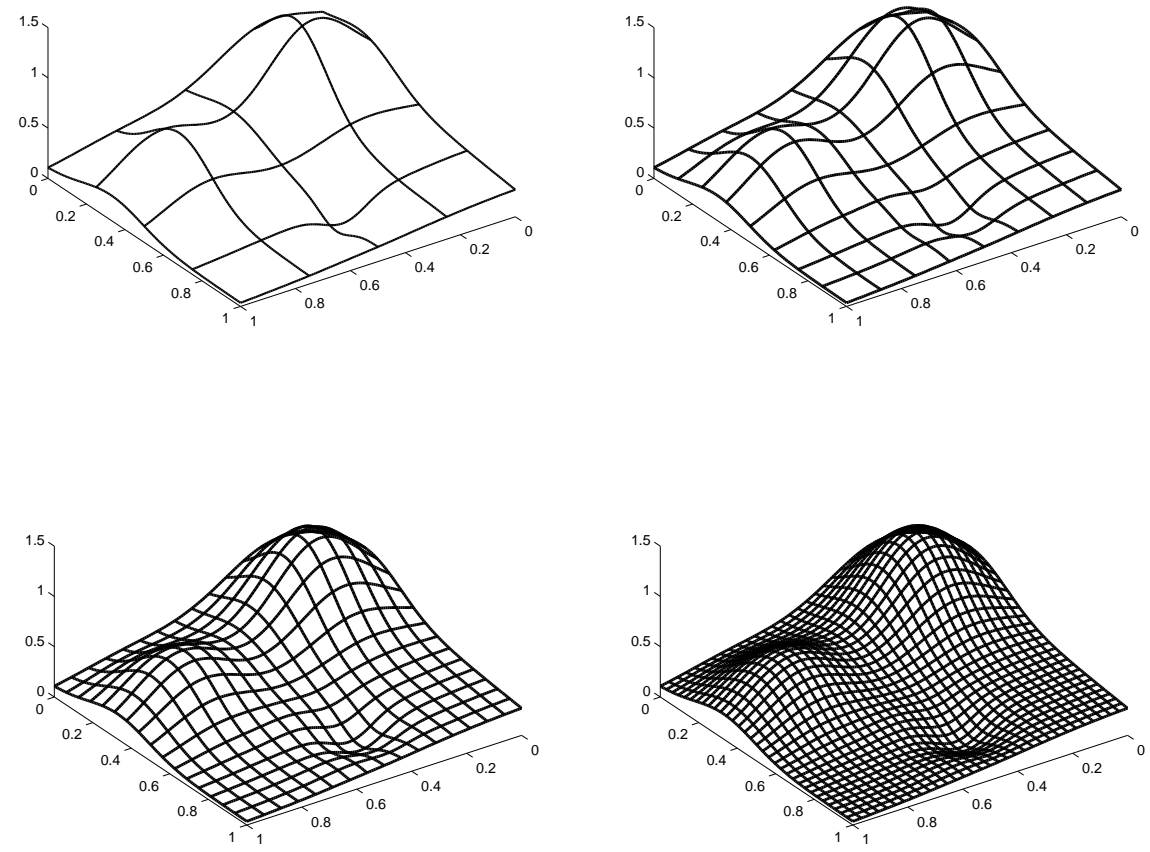

Figure 1: Example of application of an interpolatory net subdivision scheme.

\section{Interpolatory net subdivision schemes and their analysis}

In this section we give definitions and introduce some notation related to net subdivision schemes. Many of them are taken from [4] which presents general analysis tools for such schemes. In particular, 
we formulate here the convergence and smoothness results of [4] for the case of interpolatory net subdivision schemes. Also, we refer to [4, Section 5] for the definitions and the properties of subdivision schemes refining points (point subdivision schemes) relevant to our analysis.

Definition 2.1. A net $N$ is a continuous bivariate function defined on a grid of lines $T$

$$
T=T\left(d, \ell, m,\left(x_{0}, y_{0}\right)\right)=\left\{s_{i} \times\left[t_{0}, t_{\ell}\right], i=0, \cdots, m\right\} \cup\left\{\left[s_{0}, s_{m}\right] \times t_{j}, j=0, \cdots, \ell\right\},
$$

with $s_{i}=x_{0}+i d, i=0, \cdots, m$ and $t_{j}=y_{0}+j d, j=0, \cdots, \ell$, namely $N$ consists of the continuous univariate functions

$$
N\left(s, t_{j}\right), j=0, \cdots, \ell, \quad N\left(s_{i}, t\right), i=0, \cdots, m,
$$

defined on $\left[s_{0}, s_{m}\right]$ and $\left[t_{0}, t_{\ell}\right]$, respectively. We call the functions in (2) the u-functions of $N$.

If all the u-functions of a net $N$ are $C^{n}$, then the net $N$ is called $C^{n}$. The point $O=\left(x_{0}, y_{0}\right)$ is termed the origin of $T$.

To stress the relation among a net of $\mathrm{u}$-functions and the corresponding grid of lines we use the notation $N=N(T)$.

Hereinafter we also use the following notation

- $\Omega(T)$ is the convex hull of $T$;

- $\mathcal{E}(T)$ is the collection of intersection points of the grid lines of $T$

$$
\mathcal{E}(T):=\left\{\left(s_{i}, t_{j}\right), i=0, \ldots, m, j=0, \ldots, \ell\right\} ;
$$

the points of $\mathcal{E}(T)$ are termed grid points;

- $\mathcal{E}(N)=\left.N\right|_{\mathcal{E}(T)}$. (It follows from the continuity of $N$ that these points are well defined);

- the symbol $\|\cdot\|$ stands for $\|\cdot\|_{\infty}$ for vectors and for functions on their domains of definition;

- $\mathbb{N}_{0}:=\{0,1,2, \cdots\}$;

- $\mathbb{N}:=\{1,2, \cdots\}$.

We continue by introducing the grid refinement operator

$$
r(T):=T\left(\frac{d}{2}, 2 \ell, 2 m,\left(x_{0}, y_{0}\right)\right) .
$$

In the terminology of [4], where "primal" and "dual" refinements are studied, this type of grid refinement is termed "primal".

We consider in this paper a net refinement operator which is interpolatory, local, uniform and symmetric. Since near the boundaries of a grid the refinement operator cannot be applied, due to the lack of $\mathrm{u}$-functions on the other side of the boundaries, such an operator when applied to $N(T)$ generates a net defined on a refined grid which is a subset of $r(T)$, obtained by deleting a layer of boundary grid lines from $r(T)$. This leads to the following definition, with $\nu$ an integer depending on the locality of the net refinement operator.

Definition 2.2. The grid obtained from $T=T\left(d, \ell, m,\left(x_{0}, y_{0}\right)\right)$ after $T$ is cropped from the outside by deleting the outmost $\nu$ grid lines from its four sides is

$$
\operatorname{crop}_{\nu}(T):=T\left(d, \ell-2 \nu, m-2 \nu,\left(x_{0}+d \nu, y_{0}+d \nu\right)\right) .
$$

Remark 2.3. Note that the crop operator is not necessary if we introduce boundary refinement rules, as shown in [3]. 
Definition 2.4. For $T_{0}=T\left(d_{0}, \ell_{0}, m_{0},\left(x_{0}, y_{0}\right)\right)$ and an integer $\nu \geq 0$, we define the sequence of $\nu$-refined grids $\left\{T_{k}, k \in \mathbb{N}_{0}\right\}$ inductively by

$$
T_{k+1}=\operatorname{crop}_{\nu}\left(r\left(T_{k}\right)\right), \quad k \in \mathbb{N}_{0} .
$$

We can now introduce the interpolatory local net refinement operator $\mathcal{R}$ and the notion of interpolatory net subdivision scheme.

Definition 2.5. Given $N(T)$ with $d$ the grid size of $T$, let $r(T)$ be a refinement of $T$ as in (3). The operator $\mathcal{R}$ is termed an interpolatory net refinement operator if it is a local, uniform, symmetric rule for producing from a net $N(T)$ a refined net $\mathcal{R}(N)$, defined on crop $\nu(r(T))$ for some $\nu \in \mathbb{N}_{0}$, such that

$$
\left.N\right|_{\operatorname{crop}_{\nu}(r(T)) \cap T} \subset \mathcal{R}(N) .
$$

Definition 2.6. Let $\mathcal{R}$ and $\nu$ be as in Definition 2.5, and let $\left\{T_{k}, k \in \mathbb{N}_{0}\right\}$ be a sequence of $\nu$-refined grids. The iterative process

$$
\begin{aligned}
& \text { Input: } N_{0}\left(T_{0}\right) \\
& \text { For } k=0,1, \ldots, \\
& \qquad \begin{aligned}
& T_{k+1}=\operatorname{crop}_{\nu}\left(r\left(T_{k}\right)\right) \\
& N_{k+1}\left(T_{k+1}\right):=\mathcal{R}\left(N_{k}\left(T_{k}\right)\right)=\mathcal{R}^{k}\left(N_{0}\left(T_{0}\right)\right)
\end{aligned}
\end{aligned}
$$

with $\left.N_{k}\right|_{T_{k+1} \cap T_{k}} \subset N_{k+1}$, is called an interpolatory net subdivision scheme and is denoted also by $\mathcal{R}$.

In the rest of the paper we assume without loss of generality that

$$
T_{0}=T\left(d_{0}, \ell_{0}, m_{0}, O_{0}\right), \quad \text { with } \quad O_{0}=(0,0), d_{0}=1
$$

and denote $T_{k}=T\left(d_{k}, \ell_{k}, m_{k}, O_{k}\right)$ for $k \in \mathbb{N}$. Obviously $d_{k}=2^{-k}, O_{k}=O_{k-1}+\nu\left(d_{k-1}, d_{k-1}\right)$ and $\ell_{k}=2 \ell_{k-1}-2 \nu, m_{k}=2 m_{k-1}-2 \nu$.

In the following we use the term "a sequence of refined grids" also for "a sequence of $\nu$-refined grids", whenever $\nu$ is not relevant to the discussion. For a sequence of $\nu$-refined grids $\left\{T_{k}\right\}_{k \in \mathbb{N}_{0}}$ with $T_{0}$ as in (4) we denote by $\Omega_{\infty}=\Omega_{\infty}\left(T_{0}, \nu\right):=\left[2 \nu, \ell_{0}-2 \nu\right] \times\left[2 \nu, m_{0}-2 \nu\right]$ the limit of $\left\{\Omega\left(T_{k}\right)\right\}_{k \in \mathbb{N}_{0}}$ as $k \rightarrow \infty$. Also, we assume that $\ell_{0}$ and $m_{0}$ are greater than $4 \nu+1$, so that $\Omega_{\infty}$ is non-empty.

In contrast to interpolatory point subdivision schemes we cannot expect convergence of an interpolatory net subdivision scheme for any initial net. Since $\left.N_{0}\right|_{\Omega_{\infty}}$ is the limit function restricted to $T_{0} \cap \Omega_{\infty}$, the u-functions of $N_{0}$ must be at least as smooth as the limit function. So we consider initial nets in a smoothness class of nets. For $m \in \mathbb{N}_{0}$ we denote by $\mathcal{L}_{m}\left(T_{0}\right)$ the class of nets with u-functions in $C^{m}$ having Lipschitz continuous $m$-th derivatives. (The last requirement is needed for the analysis). Note that since $\Omega\left(T_{0}\right)$ is finite, $\mathcal{L}_{m+1}\left(T_{0}\right) \subset \mathcal{L}_{m}\left(T_{0}\right)$ for all $m \in \mathbb{N}_{0}$.

In the following $\mathcal{R}$ is an interpolatory net refinement operator or an interpolatory net subdivision scheme.

Definition 2.7 (Convergence). $\mathcal{R}$ is termed convergent if, for any initial net $N_{0}$ in a certain smoothness class of nets contained in $\mathcal{L}_{0}\left(T_{0}\right)$, there exists a bivariate function $F \in C\left(\Omega_{\infty}\right)$ such that the sequence of refined nets $\left\{N_{k}=\mathcal{R}^{k}\left(N_{0}\right), k \in \mathbb{N}_{0}\right\}$ satisfies

$$
F(\beta)=N_{k}(\beta), \quad \beta \in T_{k} \cap \Omega_{\infty} .
$$

The function $F$ is called the limit of the subdivision scheme and is denoted by $\mathcal{R}^{\infty}\left(N_{0}\right)$. 
Definition 2.8 ( $C^{n}$-convergence). $\mathcal{R}$ is termed $C^{n}$-convergent if it is convergent and $\mathcal{R}^{\infty}\left(N_{0}\right) \in$ $C^{n}\left(\Omega_{\infty}\right)$.

Now we introduce two important properties of sequences of refined nets generated by $\mathcal{R}$, which are central to the convergence analysis.

Definition 2.9 (Nets controlled of order 0). A sequence of refined nets $\left\{\mathcal{R}^{k}\left(N_{0}\right), k \in \mathbb{N}_{0}\right\}$ is called controlled of order 0 , if either

i) for any $k \in \mathbb{N}_{0}$ the u-functions of $N_{k}$ are Lipschitz continuous with a bound $L_{k}$ on their Lipschitz constants satisfying $\lim _{k \rightarrow \infty} 2^{-k} L_{k}=0$,

or

ii) for any $k \in \mathbb{N}_{0}$ the u-functions of $N_{k}$ have Lipschitz continuous first derivatives with a bound $L_{k}$ on their Lipschitz constants satisfying $\lim _{k \rightarrow \infty} 2^{-2 k} L_{k}=0$.

As in other papers analyzing subdivision schemes which are not linear, point subdivision schemes, we use the notion of proximity.

Definition 2.10 (Proximity of order $p$ ). $\mathcal{R}$ is in proximity of order $p>0$ with a linear, bivariate, interpolatory point refinement operator $S_{\mathbf{a}}$, if for any initial net $N_{0}$ in a certain smoothness class of nets

i) $S_{\mathbf{a}} \mathcal{E}\left(\mathcal{R}^{k}\left(N_{0}\right)\right)$ is defined on $\mathcal{E}\left(T_{k+1}\right)$;

ii) $\left\|\mathcal{E}\left(\mathcal{R}^{k+1}\left(N_{0}\right)\right)-S_{\mathbf{a}} \mathcal{E}\left(\mathcal{R}^{k}\left(N_{0}\right)\right)\right\| \leq C d_{k}^{p}$,

with $C$ a constant independent of $k$.

Theorem 2.11. Let $S_{\mathbf{a}}$ be a convergent, bivariate, interpolatory point subdivision scheme. If for any $N_{0}$ in a certain smoothness class of nets contained in $\mathcal{L}_{0}\left(T_{0}\right), \mathcal{R}$ and $S_{\mathbf{a}}$ are in proximity of order $p>0$ and $\left\{\mathcal{R}^{k}\left(N_{0}\right), k \in \mathbb{N}_{0}\right\}$ is controlled of order 0 , then $\mathcal{R}$ is convergent for any such initial net.

Note that the basic limit function of $S_{\mathrm{a}}$ in Theorem 2.11 is $L_{\infty}$-stable since it interpolates the data $\left\{\delta_{\mathbf{i}, 0}: \mathbf{i} \in \mathbb{Z}^{2}\right\}$. So Theorem 2.11 is a direct conclusion from the convergence result in [4].

To apply the smoothness results in [4] we introduce more notions, in accordance with the fact that the admissible class of initial nets is now restricted to smoother nets.

Definition 2.12 (Nets controlled of order $m \geq 1$ ). A sequence of refined nets $\left\{N_{k}=\mathcal{R}^{k}\left(N_{0}\right), k \in \mathbb{N}_{0}\right\}$ is called controlled of order $m \geq 1$, if the u-functions of $N_{k}$ have Lipschitz continuous $r^{\text {th }}$ derivatives such that either

i) for orders $r=1, \ldots, m$ there exists a bound $L_{k}$ on their Lipschitz constants, for any $k \in \mathbb{N}_{0}$, satisfying

$$
\lim _{k \rightarrow \infty} 2^{-k} L_{k}=0
$$

or

ii) for orders $r=1, \ldots, m+1$ there exists a bound $L_{k}$ on their Lipschitz constants, for any $k \in \mathbb{N}_{0}$, satisfying

$$
\lim _{k \rightarrow \infty} 2^{-2 k} L_{k}=0
$$


It is easy to see that controllability of order $m \geq 1$ implies controllability of any order $<m$.

We continue by defining inductively the classes $\mathcal{S}^{n}$ for $n \in \mathbb{N}$ of point subdivision schemes used in the smoothness analysis. $\mathcal{S}^{0}$ is the class of all convergent, bivariate, point subdivision schemes with basic limit functions that are $L_{\infty}$-stable. $\left(S_{\mathbf{a}}\right.$ in Theorem 2.11 belongs to $\left.\mathcal{S}^{0}\right)$. The class $\mathcal{S}^{n}$ is defined as the subclass of $\mathcal{S}^{n-1}$ consisting of schemes with factorizable symbols such that their divided difference schemes are in $\mathcal{S}^{n-1}$. Note that $\mathcal{S}^{n} \subset \mathcal{S}^{n-1} \subset \ldots \mathcal{S}^{1} \subset \mathcal{S}^{0}$.

Theorem 2.13. [4] Let $S_{\mathbf{a}} \in \mathcal{S}^{n}$. If for any $N_{0}$ in a certain smoothness class of nets contained in $\mathcal{L}_{m}\left(T_{0}\right), \mathcal{R}$ and $S_{\mathbf{a}}$ are in proximity of order $p>n$ and $\left\{\mathcal{R}^{k}\left(N_{0}\right), k \in \mathbb{N}_{0}\right\}$ is controlled of order $m \geq n$, then $\mathcal{R}$ is $C^{n}$-convergent for any such initial net.

\section{Interpolatory blending net subdivision schemes of Dubuc-Deslauriers type}

Our idea is to construct an interpolatory net refinement operator $\mathcal{R}$ that at each step produces a finer net of functions $N_{k+1}$ by evaluating a Gordon blending interpolant [8] to $N_{k}$ at the refined grid $T_{k+1}$. The Gordon blending interpolant we use has the form

$$
\left(\mathcal{G}_{\phi} N_{k}\right)(s, t):=\left(\mathcal{G}_{\phi}^{[s]} N_{k}\right)(s, t)+\left(\mathcal{G}_{\phi}^{[t]} N_{k}\right)(s, t)-\left(\mathcal{G}_{\phi}^{[s t]} N_{k}\right)(s, t), \quad(s, t) \in \Omega\left(T_{k}\right),
$$

where

$$
\begin{aligned}
\left(\mathcal{G}_{\phi}^{[s]} N_{k}\right)(s, t) & :=\sum_{j=0}^{\ell_{k}} N_{k}\left(s, 2^{-k} j\right) \phi\left(2^{k} t-j\right), \\
\left(\mathcal{G}_{\phi}^{[t]} N_{k}\right)(s, t) & :=\sum_{i=0}^{m_{k}} N_{k}\left(2^{-k} i, t\right) \phi\left(2^{k} s-i\right), \\
\left(\mathcal{G}_{\phi}^{[s t]} N_{k}\right)(s, t) & :=\sum_{i=0}^{m_{k}} \sum_{j=0}^{\ell_{k}} N_{k}\left(2^{-k} i, 2^{-k} j\right) \phi\left(2^{k} t-j\right) \phi\left(2^{k} s-i\right),
\end{aligned}
$$

with $\phi$ a univariate function satisfying $\phi(j)=\delta_{j, 0}, j \in \mathbb{Z}$. We term $\phi$ a blending function.

The refined net is $N_{k+1}=\left.\left(\mathcal{G}_{\phi} N_{k}\right)\right|_{T_{k+1}}$. Note that the regularity of $N_{k+1}$ is the smaller between the regularity of $\phi$ and the regularity of $N_{k}$.

For our construction of the net analogue of the Dubuc-Deslauriers $(2 n+2)$-point interpolatory subdivision scheme (hereinafter denoted by $D D_{n}$ with $n \in \mathbb{N}$ ), we use in (6) a blending function $\phi^{[n]}$ satisfying the following properties:

- $\phi^{[n]}$ is symmetric;

- $\operatorname{supp}\left(\phi^{[n]}\right)=[-(n+1),(n+1)]$;

- $\phi^{[n]} \in C^{\tilde{n}}(\mathbb{R})$ with $\tilde{n}$-th derivative Lipschitz continuous.

(We denote by $L_{\phi^{[n]}}$ the bound on all Lipschitz constants of $\left.\left(\phi^{[n]}\right)^{(\ell)}, 0 \leq \ell \leq \tilde{n}\right)$. Here $\tilde{n}=\min \left\{n, \sigma_{n}+1\right\}$ where $\sigma_{n}$ denotes the integer smoothness of the $D D_{n}$ scheme (see Table 1);

- $\phi^{[n]}$ is $\Pi_{n}$ reproducing, that is $\sum_{i \in \mathbb{Z}} i^{m} \phi^{[n]}(x-i)=x^{m}, m=0, \ldots, n$;

- $\phi^{[n]}(i)=\delta_{i, 0}, i \in \mathbb{Z}$

- $\phi^{[n]}\left(\frac{1}{2}-i\right)=a_{2 i-1}^{[n]}, i=-n, \cdots, n+1$; 
where $\left\{a_{2 i-1}^{[n]}\right\}_{i=-n}^{n+1}$ is the stencil of the insertion rule of the $D D_{n}$ subdivision scheme with mask $\mathbf{a}^{[n]}=\left\{a_{i}^{[n]}\right\}_{i=-(2 n+1)}^{2 n+1}$, given explicitly in Remark 3.4.

Two examples of piecewise polynomial functions satisfying (8)-(13) for $n=1$ can be found in [1] and [7]. In Section 5 we present a whole family of piecewise polynomial functions $\phi^{[n]}$, for $n \in \mathbb{N}$, with properties (8)-(13).

Among properties (8)-(13) the last property is less usual. It is interesting to note that property (13) holds in many cases, as proved in the following proposition.

Proposition 3.1. Assume that a function $\phi$ is symmetric, has compact support and reproduces $\Pi_{2 n}$. If in addition

$$
\phi\left(\frac{1}{2}-i\right)=0, \quad i \in \mathbb{Z}, \quad i \notin[-n, n+1]
$$

then $\phi$ satisfies

$$
\phi\left(\frac{1}{2}-i\right)=a_{2 i-1}^{[n]}, i=-n, \cdots, n+1 .
$$

Proof. If $\phi$ reproduces $\Pi_{2 n}$, then it reproduces $\left(\frac{1}{2}-x\right)^{\ell}, \ell=0,1, \cdots, 2 n$, and at $x=\frac{1}{2}$ the following equations must hold,

$$
\sum_{i \in \mathbb{Z}}\left(\frac{1}{2}-i\right)^{m} \phi\left(\frac{1}{2}-i\right)=\delta_{m, 0}, \quad m=0,1, \cdots, 2 n .
$$

These conditions, in view of (14) become a system of $2 n+1$ equations in the $2 n+2$ unknowns $\phi\left(\frac{1}{2}-i\right)$, $i=-n,-n+1, \cdots, n+1$. In fact, by the symmetry of $\phi$, there are in (15) only $n+1$ unknowns

$$
\phi\left(\frac{1}{2}+i\right), \quad i=0, \cdots, n,
$$

and all equations in (15) for odd $m$ are satisfied since by the symmetry of $\phi$ both sides of these equations equal zero.

To conclude, we have the following system of $n+1$ equations with the $n+1$ unknowns (16)

$$
\sum_{i=0}^{n}\left(\frac{1}{2}+i\right)^{2 m} \phi\left(\frac{1}{2}+i\right)=\frac{1}{2} \delta_{m, 0}, \quad m=0, \cdots, n .
$$

Now, the determinant of the system (17) is the Vandermonde $V=\operatorname{det}\left(x_{i}^{j}\right)_{i, j=0, \cdots, n}$, with $x_{i}=\left(\frac{1}{2}+i\right)^{2}$, $i=0, \cdots, n$. This determinant is non-zero because $x_{0}<x_{1}<\cdots<x_{n}$. Thus the system (17) has a unique solution, which is obtained from the values at $\frac{1}{2}+i, i=0,1, \cdots, n$ of the basic limit function $\psi^{[n]}$ of the $D D_{n}$ scheme. Indeed $\psi^{[n]}$ satisfies all the conditions of the proposition; it is symmetric with support $[-(2 n+1),(2 n+1)]$, it vanishes at $\left\{ \pm\left(n+\frac{1}{2}+i\right): i \geq 1\right\}$, and it reproduces $\Pi_{2 n}$ (by the construction of the $D D_{n}$ scheme [6]). Since $\psi^{[n]}$ satisfies (13), so does any other function satisfying the conditions of the proposition, due to the uniqueness of the solution of (17).

Remark 3.2. Note that $\phi$ satisfies (14) whenever $\operatorname{supp}(\phi)=[-(n+1),(n+1)]$.

Definition 3.3. For $\phi^{[n]}$ a blending function satisfying properties (8)-(13), the interpolatory net subdivision scheme of $D D_{n}$ type is

Input: $N_{0}\left(T_{0}\right) \in \mathcal{L}_{\tilde{n}}\left(T_{0}\right)$

For $k=0,1, \ldots$,

$$
\begin{aligned}
& T_{k+1}=\operatorname{crop}_{n+1}\left(r\left(T_{k}\right)\right) \\
& N_{k+1}=\mathcal{R}_{\phi^{[n]}}\left(N_{k}\right):=\left.\left(\mathcal{G}_{\phi^{[n]}} N_{k}\right)\right|_{T_{k+1}} .
\end{aligned}
$$


It is easy to see that, due to the definition of the Gordon blending interpolant and due to the grid refinements, the blending net subdivision scheme $\mathcal{R}_{\phi^{[n]}}$ is interpolatory. We also observe that, if $\phi^{[n]}$ is a refinable function, then the limit of the interpolatory net subdivision scheme $\mathcal{R}_{\phi^{[n]}}$ obviously exists and is the Gordon interpolant $\mathcal{G}_{\phi^{[n]}} N_{0}$. However, such a net subdivision scheme is not effective, and the interesting blending functions are not refinable. Indeed, the function $\phi^{[n]}$ of the family presented in Section 5 is not refinable for all $n \in \mathbb{N}$.

Remark 3.4. The forthcoming observations are of importance in the analysis of the schemes of Definition 3.3. This analysis is presented in the next section.

i) For a piecewise polynomial $\phi^{[n]}$, the second part of (10) follows from the first.

ii) The following explicit formulas for the Dubuc-Deslauriers masks are given in [5],

$$
a_{2 i}^{[n]}=\delta_{i, 0}, \quad a_{2 i-1}^{[n]}=\frac{n+1}{2^{4 n+1}}\left(\begin{array}{c}
2 n+1 \\
n
\end{array}\right) \frac{(-1)^{i-1}}{2 i-1}\left(\begin{array}{c}
2 n+1 \\
n+i
\end{array}\right) \quad i=-n, \cdots, n+1 .
$$

iii) Denote $A_{n}:=\sum_{i=-n}^{n+1}\left|a_{2 i-1}^{[n]}\right|$. We observed numerically that $A_{n}<2$ for $1 \leq n \leq 18$ and $A_{n}<2.5$ for $19 \leq n \leq 84$. We also conjecture that $A_{n}<4$ for all $n \in \mathbb{N}$ (see Figure 2).

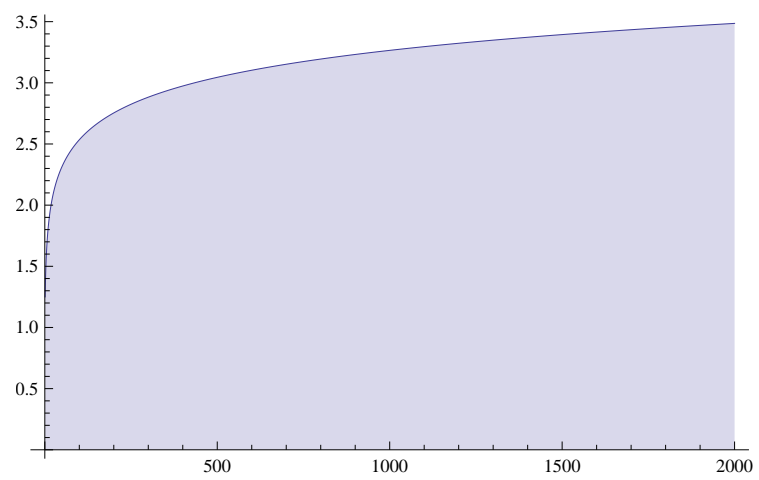

Figure 2: Plot of $A_{n}$ for $1 \leq n \leq 2000$.

iv) From [9] we know that the Hölder regularity estimate $s_{n}$ of $D D_{n}$ increases very slowly with $n$, as we can see from Table 1 (we recall that a function $\varphi$ has Hölder regularity $s_{n}=\sigma_{n}+\alpha$, $0<\alpha \leq 1$, if it is $C^{\sigma_{n}}$ and its $\sigma_{n}$-th derivative is Hölder with exponent $\alpha$ ). Moreover we observe that $\sigma_{n} \leq n$ for any $n \in \mathbb{N}, \sigma_{n} \leq n-1$ for $n \geq 5$, and $\sigma_{n} \leq n-2$ for $n \geq 7$.

\section{Convergence and smoothness analysis}

This section is devoted to the convergence and smoothness analysis of the interpolatory blending net subdivision schemes of Dubuc-Deslauriers type defined in the previous section.

For deriving convergence and smoothness we first prove that our $\mathcal{R}_{\phi^{[n]}}$ generates controlled sequences of refined nets which are in proximity with the tensor-product $D D_{n}$ scheme.

We start by proving an approximation order result for a quasi-interpolant based on a function satisfying properties (8)-(13).

Theorem 4.1. Let $x_{i}=x_{0}+i d, i \in \mathbb{N}_{0}$ and let $f$ have an $\ell$-th derivative which is Lipschitz continuous with Lipschitz constant $L_{f^{(\ell)}}$. Then for $\phi$ satisfying properties (8)-(13) with some $n$ satisfying $n \geq \ell$, the error

$$
E_{f}^{\phi}:=f-\sum_{i \in \mathbb{Z}} f\left(x_{i}\right) \phi\left(\frac{\dot{\bar{d}}}{\mathrm{~d}}-i\right)
$$




\begin{tabular}{|c|c|c|c|}
\hline$n \in \mathbb{N}$ & $\begin{array}{c}\sharp \text { of points } \\
2 n+2\end{array}$ & $\begin{array}{c}\text { Hölder regularity } \\
s_{n}\end{array}$ & $\begin{array}{c}\text { Smoothness } \\
\sigma_{n}\end{array}$ \\
\hline 1 & 4 & 2 & 1 \\
2 & 6 & 2.8300 & 2 \\
3 & 8 & 3.5511 & 3 \\
4 & 10 & 4.1935 & 4 \\
5 & 12 & 4.7767 & 4 \\
6 & 14 & 5.3173 & 5 \\
7 & 16 & 5.8294 & 5 \\
8 & 18 & 6.3233 & 6 \\
9 & 20 & 6.8054 & 6 \\
\hline
\end{tabular}

Table 1: Regularity estimates of $D D_{n}$ for $n=1, \cdots, 9$.

satisfies $\left|E_{f}^{\phi}(x)\right| \leq C_{\phi} L_{f^{(\ell)}} d^{\ell+1}$ for any $x \in \mathbb{R}$, with $C_{\phi}$ a positive constant depending only on $\phi$.

For the proof of the theorem we need a local polynomial approximation different from the Taylor polynomial.

Lemma 4.2. Under the assumptions and in the notation of Theorem 4.1 , let $P_{f}^{[j, \ell]}$ be the polynomial of degree $\ell$ interpolating $f$ at the points $x_{j}, x_{j+1}, \underbrace{x_{j+1 / 2}, \ldots, x_{j+1 / 2}}_{\ell-1}$ where $x_{j+1 / 2}:=\frac{x_{j}+x_{j+1}}{2}$.

Then the error $e_{f}^{[j, \ell]}(x):=f(x)-P_{f}^{[j, \ell]}(x)$ satisfies

$$
\left|e_{f}^{[j, \ell]}(x)\right| \leq\left(\frac{d}{2}\right)^{\ell+1} \frac{1}{\ell !} L_{f^{(\ell)}}, \quad x \in\left[x_{j}, x_{j+1}\right],
$$

and

$$
\left|e_{f}^{[j, \ell]}(x)\right| \leq(|i-j|+1)^{2}|i-j|\left(|i-j|+\frac{1}{2}\right)^{\ell-1} d^{\ell+1} \frac{1}{\ell !} L_{f^{(\ell)}}, \quad x \in\left[x_{i}, x_{i+1}\right], \quad i \neq j .
$$

Proof. Let $i$ be such that $x \in\left[x_{i}, x_{i+1}\right]$ and let $i^{*}=\min \{i, j\}$. By the error in polynomial interpolation and by the recurrence relations of divided differences,

$$
\begin{aligned}
e_{f}^{[j, \ell]}(x) & =\left(x-x_{j}\right)\left(x-x_{j+1}\right)\left(x-x_{j+1 / 2}\right)^{\ell-1}[x_{j}, x_{j+1}, \underbrace{x_{j+1 / 2}, \ldots, x_{j+1 / 2}}_{\ell-1}, x] f \\
& =\left(x-x_{j}\right)\left(x-x_{j+1}\right)\left(x-x_{j+1 / 2}\right)^{\ell-1} \frac{1}{d}\left([x_{j+1}, \underbrace{x_{j+1 / 2}, \ldots, x_{j+1 / 2}}_{\ell-1}, x] f-[x_{j}, \underbrace{x_{j+1 / 2}, \ldots, x_{j+1 / 2}}_{\ell-1}, x] f\right) \\
& =\left(x-x_{j}\right)\left(x-x_{j+1}\right)\left(x-x_{j+1 / 2}\right)^{\ell-1} \frac{1}{d} \frac{1}{\ell !}\left(f^{(\ell)}(\xi)-f^{(\ell)}(\eta)\right), \quad \xi, \eta \in\left[x_{i^{*}}, x_{i^{*}+|i-j|+1}\right] .
\end{aligned}
$$

Since $x \in\left[x_{i}, x_{i+1}\right]$, we have for $i=j$

$$
\left|x-x_{j}\right|\left|x-x_{j+1}\right| \leq\left(\frac{d}{2}\right)^{2}, \quad\left|x-x_{j+1 / 2}\right| \leq \frac{d}{2}
$$

while for $i \neq j$

$$
\left|x-x_{j}\right|\left|x-x_{j+1}\right| \leq(|i-j|+1)|i-j| d^{2}, \quad\left|x-x_{j+1 / 2}\right| \leq\left(|i-j|+\frac{1}{2}\right) d .
$$

Thus 


$$
\left|e_{f}^{[j, \ell]}(x)\right| \leq \frac{d^{\ell}}{2^{\ell+1}} \frac{1}{\ell !}\left|f^{(\ell)}(\xi)-f^{(\ell)}(\eta)\right|, \quad i=j
$$

and

$$
\left|e_{f}^{[j, \ell]}(x)\right| \leq(|i-j|+1)|i-j|\left(|i-j|+\frac{1}{2}\right)^{\ell-1} d^{\ell} \frac{1}{\ell !}\left|f^{(\ell)}(\xi)-f^{(\ell)}(\eta)\right|, \quad i \neq j .
$$

Now, since $f^{(\ell)}$ is Lipschitz continuous, we get

$$
\left|e_{f}^{[j, \ell]}(x)\right| \leq \frac{d^{\ell}}{2^{\ell+1}} \frac{1}{\ell !} L_{f^{(\ell)}} \underbrace{|\xi-\eta|}_{\leq d} \leq \frac{d^{\ell+1}}{2^{\ell+1}} \frac{1}{\ell !} L_{f^{(\ell)}}, \quad i=j
$$

and

$$
\begin{aligned}
\left|e_{f}^{[j, \ell]}(x)\right| & \leq(|i-j|+1)|i-j|\left(|i-j|+\frac{1}{2}\right)^{\ell-1} d^{\ell} \frac{1}{\ell !} L_{f^{(\ell)}} \underbrace{|\xi-\eta|}_{\leq d(|i-j|+1)} \\
& \leq(|i-j|+1)^{2}|i-j|\left(|i-j|+\frac{1}{2}\right)^{\ell-1} d^{\ell+1} \frac{1}{\ell !} L_{f^{(\ell)}}, \quad i \neq j .
\end{aligned}
$$

This concludes the proof.

We can now prove Theorem 4.1.

Proof of Theorem 4.1. Let $j$ be such that $x \in\left[x_{j}, x_{j+1}\right]$. Since $P_{f}^{[j, \ell]}$ is a polynomial of degree $\leq \ell$ then by (11) we have

$$
E_{f}^{\phi}(x)=f(x)-P_{f}^{[j, \ell]}(x)-\sum_{i \in \mathbb{Z}}\left(f\left(x_{i}\right)-P_{f}^{[j, \ell]}\left(x_{i}\right)\right) \phi\left(\frac{x}{d}-i\right) .
$$

To bound the sum in (20) we observe that by $(9) \phi\left(\frac{x}{d}-i\right)=0$, for $|i-j|>n+1$. Hence the number of terms in this sum is finite, and for $|i-j| \leq n+1$ we get from Lemma 4.2 that

$$
\left|f\left(x_{i}\right)-P_{f}^{[j, \ell]}\left(x_{i}\right)\right| \leq \tilde{C}_{\phi} L_{f^{(\ell)}} d^{\ell+1},
$$

with $\tilde{C}_{\phi}$ a constant depending on the size of the support of $\phi$. Moreover Lemma 4.2 implies that the term outside the sum in (20) is bounded by $\left(\frac{d}{2}\right)^{\ell+1} \frac{1}{\ell !} L_{f(\ell)}$. With the last two observations the desired bound is obtained from (20).

We now turn to the analysis of the net subdivision scheme $\mathcal{R}_{\phi[n]}$. The next proposition is concerned with the controllability of the generated refined nets.

Proposition 4.3. For a given $\ell \in\{1, \cdots, \tilde{n}\}$, assume that all u-functions of $N_{k}=\mathcal{R}_{\phi[n]}^{k}\left(N_{0}\right)$ have $\ell$-th derivatives which are Lipschitz continuous with Lipschitz constants bounded by $L_{k}$. Then all the u-functions of $N_{k+1}$ have $\ell$-th derivatives which are Lipschitz continuous with Lipschitz constants bounded by $L_{k+1}$ satisfying

$$
L_{k+1} \leq L_{k}\left(A_{n}+C_{\phi^{[n]}} d_{k}\right),
$$

with $C_{\phi^{[n]}}$ a positive constant depending only on $\phi^{[n]}$, and with $A_{n}$ as in Remark 3.4.

Proof. By the refinement rule (18)

$$
N_{k+1}(s, t)=\sum_{j=0}^{\ell_{k}} N_{k}\left(s, 2^{-k} j\right) \phi^{[n]}\left(2^{k} t-j\right)+\sum_{i=0}^{m_{k}}\left[N_{k}\left(2^{-k} i, t\right)-\sum_{j=0}^{\ell_{k}} N_{k}\left(2^{-k} i, 2^{-k} j\right) \phi^{[n]}\left(2^{k} t-j\right)\right] \phi^{[n]}\left(2^{k} s-i\right),
$$


with $(s, t) \in T_{k+1}$. Substituting $t=2^{-k-1} m, m \in \mathbb{Z}$, and differentiating $\ell$ times with respect to $s$ we get

$$
\begin{aligned}
& \frac{\partial^{\ell}}{\partial s^{\ell}} N_{k+1}\left(s, 2^{-k-1} m\right)=\sum_{j=0}^{\ell_{k}} \frac{\partial^{\ell}}{\partial s^{\ell}} N_{k}\left(s, 2^{-k} j\right) \phi^{[n]}\left(\frac{m}{2}-j\right) \\
&+2^{\ell k} \sum_{i=0}^{m_{k}}\left[N_{k}\left(2^{-k} i, 2^{-k-1} m\right)-\sum_{j=0}^{\ell_{k}} N_{k}\left(2^{-k} i, 2^{-k} j\right) \phi^{[n]}\left(\frac{m}{2}-j\right)\right]\left(\phi^{[n]}\right)^{(\ell)}\left(2^{k} s-i\right) .
\end{aligned}
$$

By hypothesis, $\frac{\partial^{\ell}}{\partial s^{\ell}} N_{k}\left(s, 2^{-k} j\right), j=0, \cdots, \ell_{k}$, are Lipschitz continuous with Lipschitz constants bounded by $L_{k}$. Moreover, by $(10),\left(\phi^{[n]}\right)^{(\ell)}$ is Lipschitz continuous with Lipschitz constant bounded by $L_{\phi^{[n]}}$. Now, recall that for a function of the form $f=\alpha g+\beta h$ with $g$ and $h$ Lipschitz continuous functions with Lipschitz constants $L_{g}$ and $L_{h}$ respectively, $f$ is Lipschitz continuous with Lipschitz constant $L_{f}$ satisfying $L_{f} \leq|\alpha| L_{g}+|\beta| L_{h}$. Since by (9), (12) and (13) $\sum_{j=0}^{\ell_{k}}\left|\phi^{[n]}\left(\frac{m}{2}-j\right)\right| \leq A_{n}$ for all $m \in \mathbb{Z}$, it follows that $\frac{\partial^{\ell}}{\partial s^{\ell}} N_{k+1}\left(s, 2^{-k-1} m\right)$ are Lipschitz continuous with Lipschitz constants bounded by $L_{k+1}$ satisfying

$$
L_{k+1} \leq A_{n} L_{k}+2^{\ell k} L_{\phi[n]} \sum_{i \in I_{s}}\left|E_{N_{k}\left(2^{-k} i, \cdot\right)}^{\phi^{[n]}}\left(2^{-k-1} m\right)\right|,
$$

where $A_{n}$ is as in Remark 3.4, $I_{s}=\left\{i=0, \cdots, m_{k}: 2^{k} s-i \in \operatorname{supp}\left(\phi^{[n]}\right)\right\}$ and $E_{N_{k}\left(2^{-k} i \cdot \cdot\right)}^{\phi^{[n]}}\left(2^{-k-1} m\right)$ is defined as in (19).

Since we assume that the u-functions of $N_{k}$ have Lipschitz continuous $\ell$-th derivatives with Lipschitz constants bounded by $L_{k}$, in view of Theorem 4.1 we have

$$
\left|E_{N_{k}\left(2^{-k} i, \cdot\right)}^{\phi^{[n]}}\left(2^{-k-1} m\right)\right| \leq C_{\phi^{[n]}} L_{k} d_{k}^{\ell+1} \quad \forall i \in I_{s},
$$

with $C_{\phi^{[n]}}$ a positive constant depending only on $\phi^{[n]}$. Thus by the compact support of $\phi^{[n]}$ the number of terms in the sum in (22) is bounded, and we get

$$
\sum_{i \in I_{s}}\left|E_{N_{k}\left(2^{-k} i, \cdot\right)}^{\phi^{[n]}}\left(2^{-k-1} m\right)\right| \leq C_{\phi^{[n]}} L_{k} d_{k}^{\ell+1}
$$

with $C_{\phi^{[n]}}$ a generic positive constant depending only on $\phi^{[n]}$.

Recalling that $d_{k}=2^{-k}$, we observe that the bound $L_{k+1}$ on the Lipschitz constants of the $\ell$-th derivatives of the net functions at level $k+1$ is bounded as follows

$$
L_{k+1} \leq A_{n} L_{k}+C_{\phi[n]} L_{k} d_{k}=L_{k}\left(A_{n}+C_{\phi[n]} d_{k}\right),
$$

which asserts the claim of the proposition.

Proposition 4.4. Under the conditions of Proposition 4.3,

(i) if $A_{n} \in[1,2)$ then there exists $\beta \in(0,1)$ and $M>0$ such that $L_{k} d_{k}^{\beta} \leq M$ for $k \in \mathbb{N}_{0}$;

(ii) if $A_{n} \in[2,4)$ then there exists $\beta \in(1,2)$ and $M>0$ such that $L_{k} d_{k}^{\beta} \leq M$ for $k \in \mathbb{N}_{0}$.

Proof. Observe that in both cases (i) and (ii) $\beta$ can be chosen such that $2^{\beta}>A_{n}$ (more precisely, in case (i) $\beta$ is in $(0,1)$ while in case (ii) it is in $(1,2))$.

Then, by Proposition 4.3 we have

$$
L_{k+1} \leq L_{k}\left(A_{n}+C_{\phi^{[n]}} d_{k}\right)
$$


Multiplying by $d_{k+1}^{\beta}=d_{k}^{\beta} 2^{-\beta}$, we get

$$
L_{k+1} d_{k+1}^{\beta} \leq L_{k} d_{k}^{\beta}\left(\frac{A_{n}}{2^{\beta}}+C_{\phi^{[n]}} \frac{d_{k}}{2^{\beta}}\right) .
$$

By the choice of $\beta$, there exists $k^{*}$ large enough such that $\frac{A_{n}}{2^{\beta}}+C_{\phi^{[n]}} 2^{-k^{*}-\beta}=1$. Then, for all $k>k^{*}$, $\frac{A_{n}}{2^{\beta}}+C_{\phi[n]} 2^{-k-\beta}<1$, and

$$
L_{k+1} d_{k+1}^{\beta} \leq L_{k} d_{k}^{\beta} \leq \cdots \leq L_{k^{*}} d_{k^{*}}^{\beta} .
$$

The claim of the proposition follows by choosing $M=\max _{1 \leq j \leq k^{*}} L_{j} d_{j}^{\beta}$.

For the next result we note that by its definition and by Table $1 \tilde{n}=\min \left\{n, \sigma_{n}+1\right\} \geq 1$ for all $n \in \mathbb{N}$.

Corollary 4.5. The net subdivision scheme $\mathcal{R}_{\phi[n]}$ when operating on $N_{0} \in \mathcal{L}_{m}\left(T_{0}\right)$ with $m \in[1, \tilde{n}] \cap \mathbb{Z}$, generates sequences of refined nets $\left\{\mathcal{R}_{\phi[n]}^{k}\left(N_{0}\right), k \in \mathbb{N}_{0}\right\}$ which are controlled of order $m$ if $A_{n} \in[1,2)$ and controlled of order $m-1$ if $A_{n} \in[2,4)$.

Proof. Denote by $L_{k}$ the bound on the Lipschitz constants of the derivatives up to order $m$ of all the u-functions of $\mathcal{R}_{\phi[n]}^{k}\left(N_{0}\right)$. If $A_{n} \in[1,2)$ then it follows from case (i) of Proposition 4.4 that $\beta \in(0,1)$ and that $\lim _{k \rightarrow \infty} L_{k} d_{k}=0$. Therefore by the definition of controllability (Definition 2.12, case i)) $\mathcal{R}_{\phi[n]}$ generates sequences of refined nets which are controlled of order $m$, whenever $N_{0} \in \mathcal{L}_{m}\left(T_{0}\right)$.

Analogously, if $A_{n} \in[2,4), \beta \in(1,2)$ by case (ii) of Proposition 4.4 and $\lim _{k \rightarrow \infty} L_{k} d_{k}^{2}=0$. Therefore by the definition of controllability (Definition 2.12, case ii)) $\mathcal{R}_{\phi^{[n]}}$ generates sequences of refined nets which are controlled of order $m-1$, whenever $N_{0} \in \mathcal{L}_{m}\left(T_{0}\right)$.

Since controllability of order 1 implies controllability of order 0 , we have

Corollary 4.6. The net subdivision scheme $\mathcal{R}_{\phi[n]}$, for any $n \leq 84$, generates sequences of refined nets $\left\{\mathcal{R}_{\phi[n]}^{k}\left(N_{0}\right), k \in \mathbb{N}_{0}\right\}$ which are controlled of order 0 , whenever $N_{0} \in \mathcal{L}_{1}\left(T_{0}\right)$.

We turn now to the proximity of $\mathcal{R}_{\phi[n]}$ with the tensor-product $D D_{n^{-}}$-scheme.

Proposition 4.7. $\mathcal{R}_{\phi^{[n]}}$ as in (18) with $\phi^{[n]}$ satisfying (8)-(13), is in proximity of order $p=\tilde{n}+1-\beta>$ 0 with the tensor-product $D D_{n}$ scheme, for any $N_{0} \in \mathcal{L}_{\tilde{n}}\left(T_{0}\right)$.

Proof. It is easy to see that for $S_{n}$-the tensor-product $D D_{n}$ scheme, $S_{n} \mathcal{E}\left(N_{k}\right)$ is defined over $\mathcal{E}\left(T_{k+1}\right)$. Now let $N_{k}:=\mathcal{R}_{\phi[n]}^{k}\left(N_{0}\right)$, and consider $e(\mathbf{q})=N_{k+1}\left(2^{-k-1} \mathbf{q}\right)-\left(S_{n} \mathcal{E}\left(N_{k}\right)\right)\left(2^{-k-1} \mathbf{q}\right)$ for any $\mathbf{q}=$ $(r, m) \in \mathbb{Z}^{2}$. Since by $(9),(12),(13) \phi^{[n]}$ coincides with the basic limit function of $D D_{n}$ at $\frac{1}{2} \mathbb{Z}$, and since $\left.\left(S_{n} \mathcal{E}\left(N_{k}\right)\right)\right|_{T_{k+1}}=\left.\left(S_{n}^{\infty} \mathcal{E}\left(N_{k}\right)\right)\right|_{T_{k+1}}$, we have

$$
\left(S_{n} \mathcal{E}\left(N_{k}\right)\right)\left(2^{-k-1} \mathbf{q}\right)=\sum_{i=0}^{m_{k}} \sum_{j=0}^{\ell_{k}} N_{k}\left(2^{-k} i, 2^{-k} j\right) \phi^{[n]}\left(\frac{m}{2}-j\right) \phi^{[n]}\left(\frac{r}{2}-i\right) .
$$

Thus 


$$
\begin{aligned}
e(\mathbf{q}) & =\sum_{j=0}^{\ell_{k}} N_{k}\left(2^{-k-1} r, 2^{-k} j\right) \phi^{[n]}\left(\frac{m}{2}-j\right) \\
& +\sum_{i=0}^{m_{k}}\left[N_{k}\left(2^{-k} i, 2^{-k-1} m\right)-\sum_{j=0}^{\ell_{k}} N_{k}\left(2^{-k} i, 2^{-k} j\right) \phi^{[n]}\left(\frac{m}{2}-j\right)\right] \phi^{[n]}\left(\frac{r}{2}-i\right) \\
& -\sum_{i=0}^{m_{k}} \sum_{j=0}^{\ell_{k}} N_{k}\left(2^{-k} i, 2^{-k} j\right) \phi^{[n]}\left(\frac{m}{2}-j\right) \phi^{[n]}\left(\frac{r}{2}-i\right) \\
& =\sum_{j=0}^{\ell_{k}}\left[N_{k}\left(2^{-k-1} r, 2^{-k} j\right)-\sum_{i=0}^{m_{k}} N_{k}\left(2^{-k} i, 2^{-k} j\right) \phi^{[n]}\left(\frac{r}{2}-i\right)\right] \phi^{[n]}\left(\frac{m}{2}-j\right) \\
& +\sum_{i=0}^{m_{k}}\left[N_{k}\left(2^{-k} i, 2^{-k-1} m\right)-\sum_{j=0}^{\ell_{k}} N_{k}\left(2^{-k} i, 2^{-k} j\right) \phi^{[n]}\left(\frac{m}{2}-j\right)\right] \phi^{[n]}\left(\frac{r}{2}-i\right) \\
& =\sum_{j=0}^{\ell_{k}} E_{N_{k}\left(\cdot, 2^{-k} j\right)}^{\phi^{[n]}}\left(2^{-k-1} r\right) \phi^{[n]}\left(\frac{m}{2}-j\right)+\sum_{i=0}^{m_{k}} E_{N_{k}\left(2^{-k} i, \cdot\right)}^{\phi^{[n]}}\left(2^{-k-1} m\right) \phi^{[n]}\left(\frac{r}{2}-i\right) .
\end{aligned}
$$

In view of Theorem 4.1, and since for $N_{0} \in \mathcal{L}_{\tilde{n}}\left(T_{0}\right)$ we have by Proposition 4.3 that the u-functions of $N_{k}$ have Lipschitz continuous $\tilde{n}$-th derivatives with Lipschitz constants bounded by $L_{k}$ satisfying (21), we get

$$
|e(\mathbf{q})| \leq C_{\phi^{[n]}} L_{k} d_{k}^{n+1}\left(\sum_{j=0}^{\ell_{k}}\left|\phi^{[n]}\left(\frac{m}{2}-j\right)\right|+\sum_{i=0}^{m_{k}}\left|\phi^{[n]}\left(\frac{r}{2}-i\right)\right|\right) .
$$

Moreover, by Proposition $4.4 L_{k} d_{k}^{\beta} \leq M$, and we have

$$
|e(\mathbf{q})| \leq C_{\phi^{[n]}} M d_{k}^{\tilde{n}+1-\beta}\left(\sum_{j=0}^{\ell_{k}}\left|\phi^{[n]}\left(\frac{m}{2}-j\right)\right|+\sum_{i=0}^{m_{k}}\left|\phi^{[n]}\left(\frac{r}{2}-i\right)\right|\right) \leq 2 A_{n} C_{\phi^{[n]}} M d_{k}^{\tilde{n}+1-\beta} .
$$

The claim is obtained by noting that $2 A_{n} C_{\phi[n]} M$ is a constant independent of $k$.

By Proposition 4.7, $\mathcal{R}_{\phi[n]}$ for $n \leq 84$ is in proximity of order $p>0$ with $S_{n}$-the tensor-product $D D_{n}$ scheme- whenever $N_{0} \in \mathcal{L}_{\tilde{n}}\left(T_{0}\right)$. Also, by Corollary $4.6\left\{\mathcal{R}_{\phi^{[n]}}^{k}\left(N_{0}\right), k \in \mathbb{N}_{0}\right\}$ for $n \leq 84$ is controlled of order 0 for any $N_{0} \in \mathcal{L}_{1}\left(T_{0}\right)$. Thus, we conclude from Theorem 2.11 that

Corollary 4.8. For any $n \leq 84$ and for any initial net $N_{0} \in \mathcal{L}_{\tilde{n}}\left(T_{0}\right)$, the subdivision scheme $\mathcal{R}_{\phi[n]}$ converges.

Moreover, for $n \leq 84$ the proximity of order $\tilde{n}+1-\beta>0$ of $\mathcal{R}_{\phi^{[n]}}$ with $S_{n}$ for any $N_{0} \in \mathcal{L}_{\tilde{n}}\left(T_{0}\right)$, proved in Proposition 4.7, and the controllability of order $\tilde{n}$ or $\tilde{n}-1$ of $\left\{\mathcal{R}_{\phi[n]}^{k}\left(N_{0}\right), k \in \mathbb{N}_{0}\right\}$ for such $N_{0}$, proved in Corollary 4.5, together with $S_{n} \in \mathcal{S}_{\sigma_{n}}$, and Theorem 2.13 lead to the following result.

Corollary 4.9. For any $n \leq 84$ the smoothness of the refinement scheme $\mathcal{R}_{\phi^{[n]}}$ is $\sigma_{n}$.

Proof. Recall that $\tilde{n}=\min \left\{n, \sigma_{n}+1\right\}$ with $\sigma_{n}$ the integer smoothness of the $D D_{n}$ scheme. Let $\sigma_{\mathcal{R}_{\phi}[n]}$ denote the smoothness of $\mathcal{R}_{\phi[n]}$. For $1 \leq n \leq 18, \beta \in(0,1)$, and we have $\sigma_{\mathcal{R}_{\phi}[n]}=\min \left\{\sigma_{n}, \tilde{n},\lceil\tilde{n}+1-\right.$ $\beta\rceil\}=\min \left\{\sigma_{n}, \tilde{n}\right\}=\sigma_{n}$. In case $19 \leq n \leq 84, \quad \beta \in(1,2)$ and $\sigma_{\mathcal{R}_{\phi}[n]}=\min \left\{\sigma_{n}, \tilde{n}-1,\lceil\tilde{n}+1-\beta\rceil\right\}=$ $\min \left\{\sigma_{n}, \tilde{n}-1\right\}=\sigma_{n}$, since for $n \geq 7, \sigma_{n} \leq n-2$ (see Table 1) and therefore $\tilde{n}=\sigma_{n}+1$.

In view of part (iii) of Remark 3.4, we conjecture that Corollaries 4.8 and 4.9 hold for any $n \in \mathbb{N}$. 


\section{A family of interpolatory blending net subdivision schemes of Dubuc-Deslauriers type}

A specific example of a family of interpolatory blending net subdivision schemes of Dubuc-Deslauriers type is considered in this section. It is based on a family of functions satisfying properties (8)-(13) of Section 3, which is known in the literature under the name Z-splines $[2,10]$. These are the cardinal piecewise polynomial functions with integer knots presented in the next definition.

Definition 5.1 (Z-splines). Let $n \in \mathbb{N}$. We denote by $Z^{[n]}$ the piecewise polynomial function of degree $2 n+1$ with integer break points, satisfying:

(i) $Z^{[n]}$ is symmetric;

(ii) $\operatorname{supp}\left(Z^{[n]}\right)=[-(n+1),(n+1)]$;

(iii) $Z^{[n]} \in C^{n}(\mathbb{R})$;

(iv) $\sum_{i \in \mathbb{Z}} i^{m} Z^{[n]}(x-i)=x^{m}, m=0,1, \cdots, 2 n$;

(v) $Z^{[n]}(i)=\delta_{i, 0}, i \in \mathbb{Z}$.

The existence and uniqueness of $Z^{[n]}$ is shown in $[2,10]$. Definition 5.1 implies that properties (8)-(12) are satisfied by $Z^{[n]}$. Property (13) is obtained in view of (ii) and (iv) in Definition 5.1 and Proposition 3.1. Thus, since $Z^{[n]}$ has properties (8)-(13), the blending net subdivision scheme based on it is convergent and its limit function has the same integer smoothness as that of the corresponding $D D_{n}$ scheme.

In the following we show several numerical examples generated by the interpolatory net subdivision schemes of Dubuc-Deslauriers type, based on $Z^{[1]}$ and $Z^{[2]}$. The $Z^{[1]}$ spline has support $[-2,2]$ and is given on $[0,2]$ by

$$
Z^{[1]}(x)= \begin{cases}1-\frac{5}{2} x^{2}+\frac{3}{2} x^{3}, & 0 \leq x<1 \\ 2-4 x+\frac{5}{2} x^{2}-\frac{1}{2} x^{3}, & 1 \leq x<2 .\end{cases}
$$

It is defined on $[-2,0]$ by symmetry. Figure 3 left presents the graph of $Z^{[1]}$. In view of Corollary 4.9 the corresponding blending net subdivision scheme produces $C^{1}$ limit functions whenever $N_{0} \in \mathcal{L}_{1}\left(T_{0}\right)$. Since $\operatorname{supp}\left(Z^{[1]}\right)=[-2,2]$, we get for this scheme that $T_{k+1}=\operatorname{crop}_{2}\left(r\left(T_{k}\right)\right)$. The result of the application of three iterations of the interpolatory net subdivision scheme based on $Z^{[1]}$ (the result is defined on $T_{3}$ ) is shown in Figure 4.

We continue with the $Z^{[2]}$ spline, supported on $[-3,3]$. On $[0,3]$ it is given by

$$
Z^{[2]}(x)= \begin{cases}1-\frac{15}{12} x^{2}-\frac{35}{12} x^{3}+\frac{63}{12} x^{4}-\frac{25}{12} x^{5}, & 0 \leq x<1, \\ -4+\frac{75}{4} x-\frac{245}{8} x^{2}+\frac{545}{24} x^{3}-\frac{63}{8} x^{4}+\frac{25}{24} x^{5}, & 1 \leq x<2, \\ 18-\frac{153}{4} x+\frac{255}{8} x^{2}-\frac{313}{24} x^{3}+\frac{21}{8} x^{4}-\frac{5}{24} x^{5}, & 2 \leq x<3,\end{cases}
$$

and on $[-3,0]$ it is defined by symmetry. Figure 3 right shows the graph of $Z^{[2]}$. In view of Corollary 4.9 , the blending net subdivision scheme based on $Z^{[2]}$ is $C^{2}$ whenever $N_{0} \in \mathcal{L}_{2}\left(T_{0}\right)$. The result of the application of three iterations of this net subdivision scheme is shown in Figure 5. Note that for this scheme $T_{k+1}=\operatorname{crop}_{3}\left(r\left(T_{k}\right)\right)$, and therefore $T_{3}$ in Figure 5 is smaller than $T_{3}$ in Figure 4. 

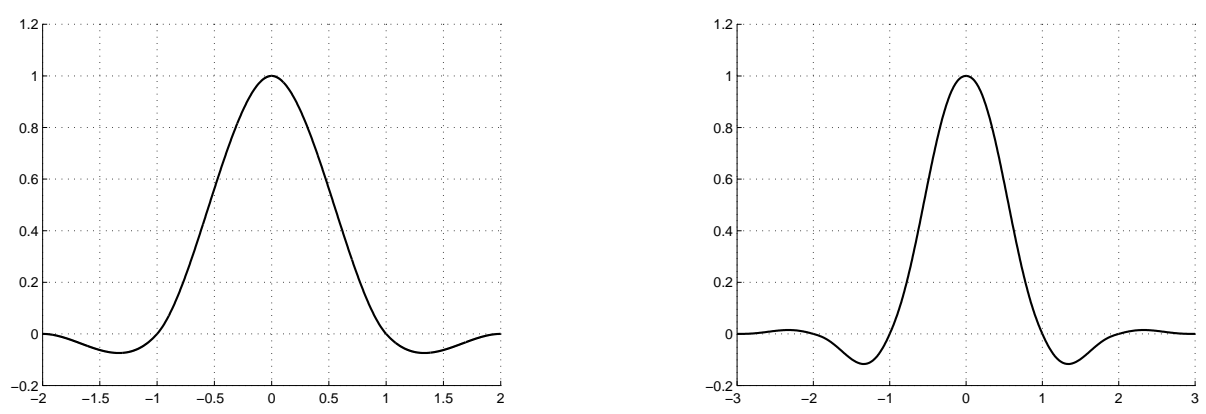

Figure 3: Plot of the cardinal basis function $Z^{[1]}$ (left) and $Z^{[2]}$ (right).

\section{References}

[1] C. Beccari, G. Casciola, L. Romani, Non-uniform interpolatory curve subdivision with edge parameters built upon compactly supported fundamental splines, BIT Numer. Math. 51(4), 781-808 (2011).

[2] J.T. Becerra Sagredo, Z-splines: moment conserving cardinal spline interpolation of compact support for arbitrarily spaced data. Research report no. 2003-10, Eidgenössische Technische Hochschule, Zurich, Switzerland, 2003.

[3] C. Conti, N. Dyn, Blending based corner-cutting subdivision scheme for nets of curves, Comput. Aided Geom. Design 27(4), 340-358 (2010).

[4] C. Conti, N. Dyn, Analysis of subdivision schemes for nets of functions by proximity and controllability, J. Comp. Appl. Math. 236(4), 461-475 (2011).

[5] J.M. de Villiers, K.M. Goosen, B.M. Herbst, Dubuc-Deslauriers subdivision for finite sequences and interpolation wavelets on an interval, SIAM J. Math. Anal. 35, 423-452 (2003).

[6] G. Deslauriers, S. Dubuc, Symmetric iterative interpolation processes, Constr. Approx. 5, 49-68 (1989).

[7] O. Elisha, Interpolatory refinements of nets of curves, Master Thesis, Tel Aviv University, Israel, 2011.

[8] W.J. Gordon, Spline-blended surface interpolation through curve networks, J. of Mathematics and Mechanics 18(10), 931-952 (1969).

[9] O. Rioul, Simple Regularity criteria for subdivision schemes, SIAM J. Math. Anal. 23(6), 15441576 (1992).

[10] T. Ueno, S. Truscott, M. Okada, New spline basis functions for sampling approximations, Numer. Algor. 45, 283-293 (2007). 

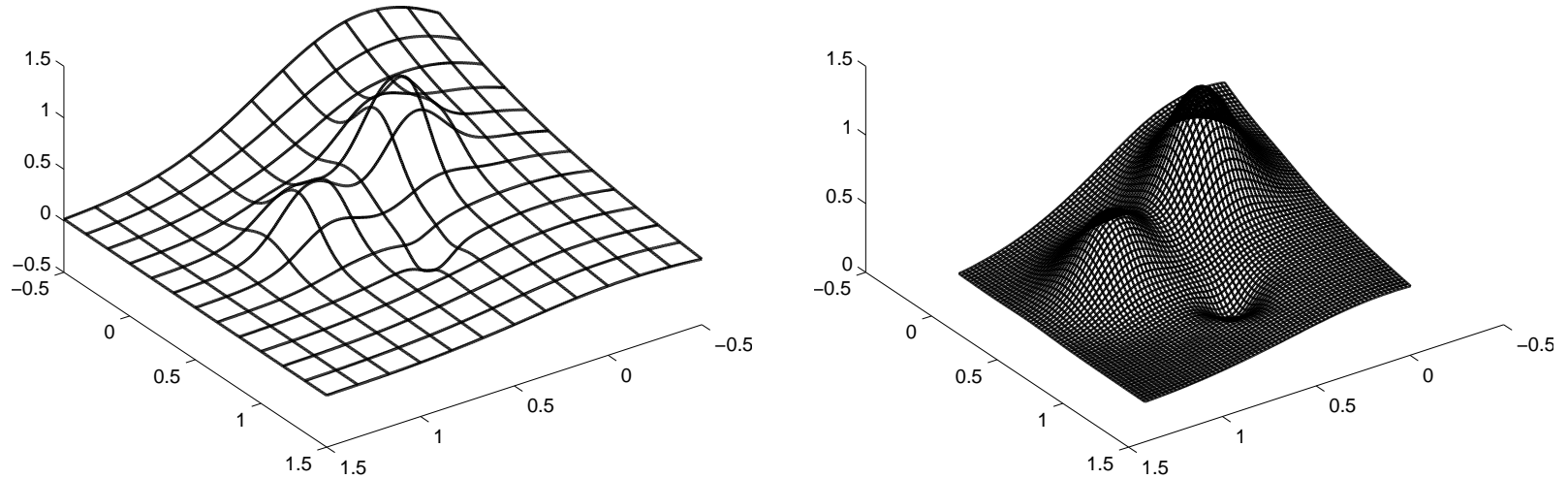

Starting net of functions

Refinement step $\mathrm{k}=3$
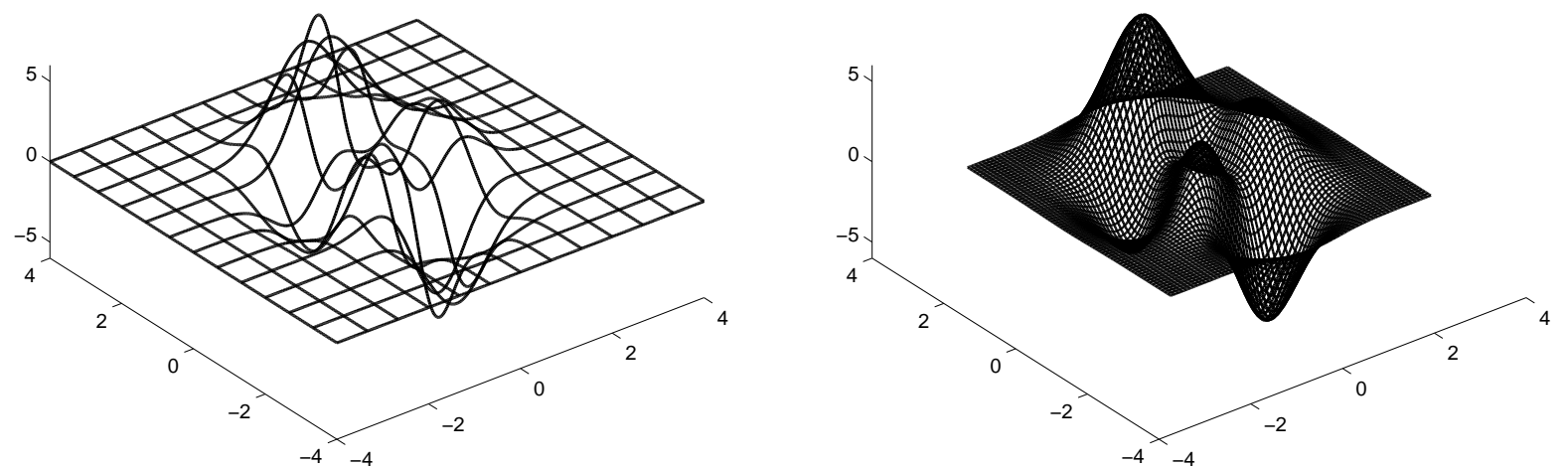

Figure 4: Three refinement steps of the net subdivision scheme based on $Z^{[1]}$. The initial net of u-functions is sampled from Franke's test function (first row) and from Matlab Peaks function (second row). First column: original net of u-functions; second column: net of u-functions defined on $T_{3}$. 

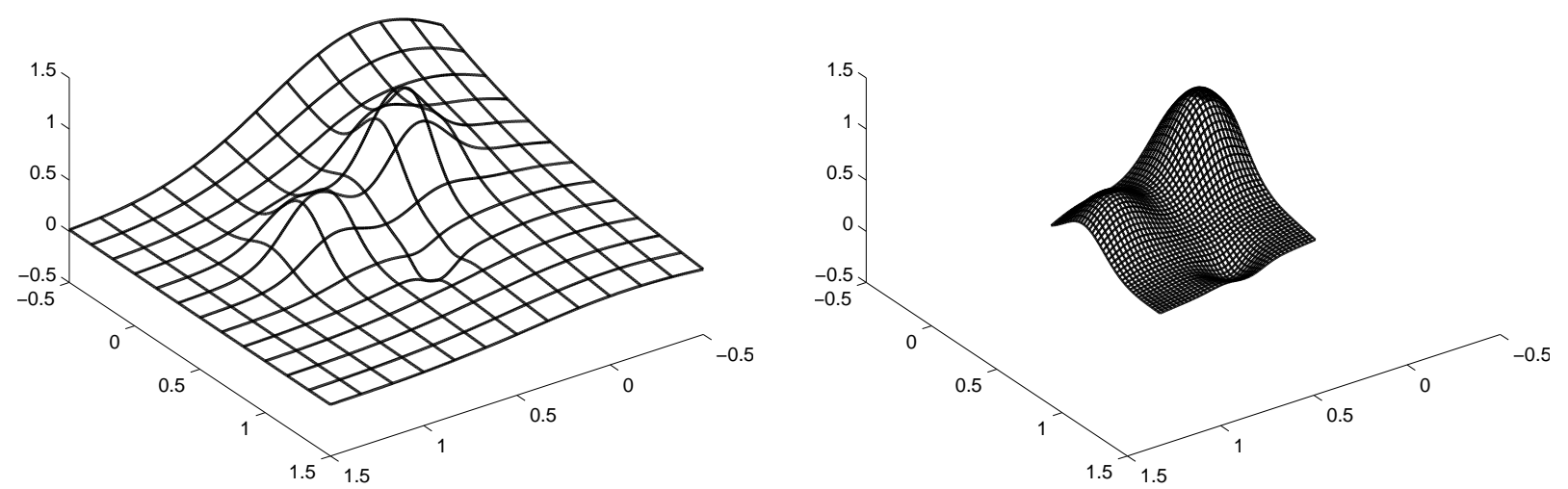

Starting net of functions

Refinement step k=3
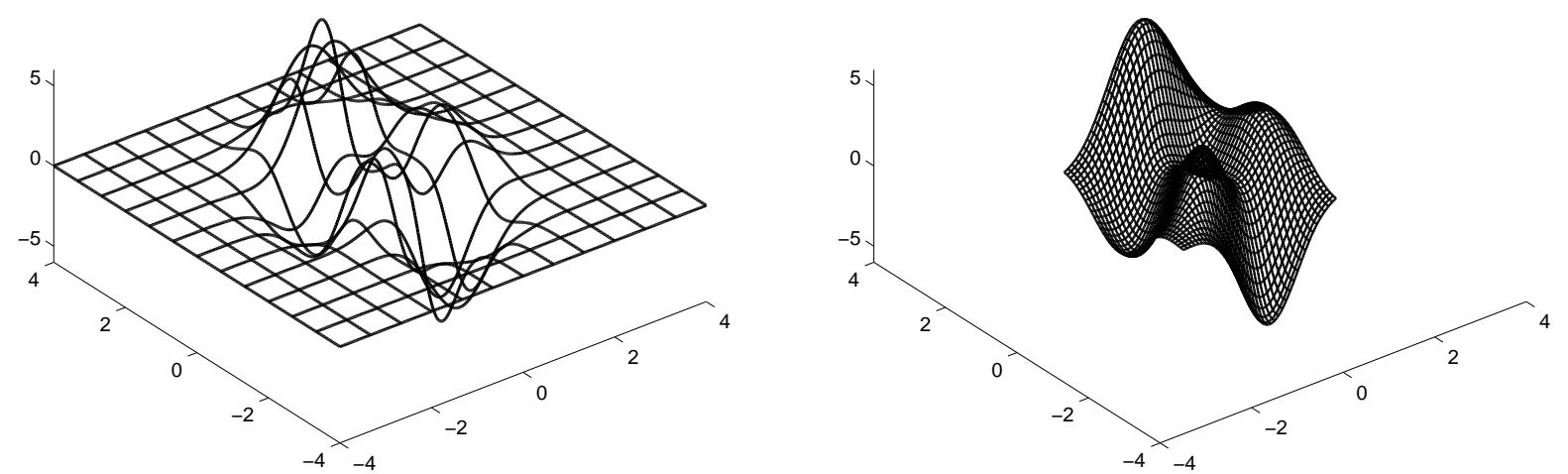

Figure 5: Three refinement steps of the net subdivision scheme based on $Z^{[2]}$. The initial net of u-functions is sampled from Franke's test function (first row) and from Matlab Peaks function (second row). First column: original net of u-functions; second column: net of u-functions defined on $T_{3}$. 